\title{
SEMIGROUP IDEALS IN RINGS
}

\author{
DAVID A. HILL
}

(Received 22 April 1974, revised 10 June 1978)

Communicated by T. E. Hall

\begin{abstract}
A ring $R$ is called an $l$-ring ( $r$-ring) in case $R$ contains an indentity and every left (right) semigroup ideal is a left (right) ring ideal. A number of structure theorems are obtained for $l$-rings when $R$ is left noetherian and left artiniar. It is shown that left noetherian $l$-rings are local left principal ideal rings. When $R$ is a finite dimensional algebra over a field, the property of being an $l$-ring is equivalent to being an $r$-ring. However, examples are given to show that these two concepts are in general not equivalent even in the artinian case.
\end{abstract}

Subject classification (Amer. Math. Soc. (MOS) 1970): 16 A 46, 16 A 48, 20 M 10.

\section{Introduction}

By definition any left ideal $I$ in a ring $(R,+,$.$) is a left ideal of the semigroup$ $(R,$.$) . We shall call the former left ring ideals and the latter left semigroup ideals$ of $R$. We consider the problem of determining when a left semigroup ideal of a ring is a left ring ideal, and note that not even $Z$, the ring of integers, has this property. Hence the problem is a nontrivial one and was first considered by Gluskin (1960). He was able to show that $R$ possesses a number of properties whenever every left semigroup ideal is a left ring ideal. Among these properties are that $R$ must be local.

Following the notation of Gluskin, we shall say that a ring $R$ is an $l$-ring ( $r$-ring) in case $R$ contains an identity and every left (right) semigroup ideal in $R$ is a left (right) ring ideal. The property of being an $l$-ring imposes strong constraints on the left ideal structure of $\boldsymbol{R}$. For example, $l$-rings have their principal left ideals linearly ordered by set theoretic inclusion (in fact this property is equivalent to being an l-ring).

In Section 1 we prove a number of general results on semigroup ideals in rings, and consider the structure of noetherian l-rings. It is shown that left noetherian $l$-rings are local left principal ideal rings. 
In Section 2 the structure of artinian $l$-rings is studied. For finite dimensional algebras over fields, the property of being an $l$-ring and of being an $r$-ring are equivalent. This means that for local finite dimensional algebras over fields left generalized uniserial is the same as generalized uniserial. Section 3 is devoted to the construction of an example of a non-noetherian $l$-ring.

We shall use the following notation throughout. The ring $R$ is associative with unity. We let $J$ denote the Jacobson radical, and ${ }_{R} M\left(M_{R}\right)$ signifies that $M$ is a left (right) $R$-module. All unspecified one-sided properties of a ring are presumed to hold on both sides, for example, an artinian (noetherian) ring is both a left and right artinian (noetherian) ring.

The author wishes to thank the referee for his kind advice and suggestions.

\section{Semigroup ideals}

Any set $S$ is linearly ordered in case there is an order $\leqslant$ on $S$ such that for $\alpha, \beta \in S, \alpha \leqslant \beta$ or $\beta \leqslant \alpha$. Consider the set of principal left ideals of $R$ partially ordered by inclusion. The following proposition is the key to most of the results which follow. It shows that $l$-rings possess the characterizing property that their principal left ideals are linearly ordered by inclusion. The proof is similar to that given by Gluskin (1960).

1.1 Proposition. Let $R$ be a ring. The following statements are equivalent:

(1) $R$ is an l-ring.

(2) $R$ has the property that for any two elements $x, y \in R R x \subseteq R y$ or $R y \subseteq R x$, that is, principal left ideals are linearly ordered by inclusion.

Proof $\Rightarrow$ Suppose $R$ is an $l$-ring. Let $x, y \in R$ and consider $S=R x \cup R y$. Then $S$ is a left semigroup ideal. Since $R$ is an $l$-ring $S$ is a left ideal and so $x+y \in S$. So $x+y \in R x$ or $x+y \in R y$. Suppose $x+y \in R x$; hence $x+y=\alpha x$. Thus $y=(\alpha-1) x$ which implies $R y \subseteq R x$.

$\Leftarrow$ Let $S$ be a left semigroup ideal. For $x, y \in S$, we have $R x \subseteq R y$ or $R y \subseteq R x$. Suppose $R x \subseteq R y$. Then $x=\alpha y$ so that

$$
x+y=\alpha y+y=(\alpha+1) y \in S .
$$

Thus $S$ is a left ring ideal.

The following corollary is due to Gluskin (1960).

1.2 Corollary. Every l-ring is a local ring.

1.3 COROLlaRY. Let $R$ be an l-ring. Then every finitely generated left ideal is principal. 
Proof. Let $I$ be a finitely generated left ideal generated by $x_{1}, \ldots, x_{n}$. By 1.1 , the principal left ideals generated by each $x_{i}$ are linearly ordered say

$$
R x_{n} \subseteq R x_{n-1} \subseteq \ldots \subseteq R x_{1} .
$$

Hence $I=R x_{1}$. Thus from Corollary 1.3 it can easily be seen that if $R$ is a left noetherian l-ring, all left ideals must be principal.

1.4 Lemma. Suppose $R$ is noetherian. If $R$ is an l-ring then,

(1) $\bigcap_{n=1}^{\infty} J^{n}=0$ and $J^{k} \supset J^{k+1} \neq 0$ for all $k \geqslant 1$, or

(2) $\bigcap_{n=1}^{\infty} J^{n}=0$ and $R$ is artinian with $J^{k}=0$ for some $k \geqslant 1$.

Proof. That $\bigcap_{\infty}^{n=1} J^{n}=0$ follows easily from 1.3 and Lemma 3.1 of Goldie(1962). If $R$ is artinian (2) follows from well-known properties of artinian rings, and Nakayama's lemma. Otherwise (1) holds using another application of Nakayama's lemma.

For noetherian $l$-rings it is now possible to determine their left ideals.

1.5 LeMMA. Let $R$ be a noetherian l-ring. Then the nonzero left ideals of $R$ are the $J^{n}, n \geqslant 0\left(\right.$ here $\left.J^{0}=R\right)$.

Proof. Let $I \neq 0$ be a left ideal in $R$. Hence $I=R x$ for some $x \in R$. By 1.4, $x \in J^{n-1}, x \notin J^{n}$ for some $n>0$. Hence by $1.1 R x \supset J^{n}$ since $J^{n}$ is principal. Let $J^{n-1}=R y$. Hence $J^{n-1} / J^{n}=R y / J y$. Define a map $\varphi: R / J \rightarrow J^{n-1} / J^{n}$ via $\varphi(\alpha+J)=$ $\alpha y+J y(\alpha \in R)$.

It is routine to check that $\varphi$ in fact defines an isomorphism between $J^{n-1} / J^{n}$ and $R / J$. By the same argument $R x / J^{n} \cong R / J$. This easily implies that $J^{n-1}=R x=I$.

The following theorem serves to characterize noetherian $l$-rings.

1.6 THEOREM. Let $R$ be noetherian. The following statements are equivalent

(a) $R$ is an l-ring.

(b) The nonzero left ideals of $R$ are precisely the $J^{n}, n \geqslant 0$, where each $J^{n}$ is cyclic.

(c) $R$ satisfies the following properties:

(1) $R$ is local.

(2) $J^{n} / J^{n+1}$ is simple as a left $R$-module for all $n \geqslant 0$ such that $J^{n} \neq 0$.

(3) $\bigcap_{n=1}^{\infty} J^{n}=0$.

Proof. (a) $\Rightarrow$ (b). This follows from 1.3 and 1.5.

(b) $\Rightarrow$ (c). $R$ must be local for (b) implies that $J$ is the unique maximal ideal. Now since each ${ }_{R} J^{n}$ is cyclic, $J^{n} \neq J^{n+1}$ whenever $J^{n} \neq 0$. Thus $J^{n} / J^{n+1} \cong R / J$ as a left $R$-module, and hence is simple. Now $\bigcap_{n=1}^{\infty} J^{n}=0$. For if $0 \neq R a \subset \bigcap_{n=0}^{\infty} J^{n}$ then by 
(b) $R a=J^{k}=\bigcap_{n=1}^{\infty} J^{n}$, that is $J^{k}=J^{k+1} \neq 0$ for some $k>0$ which contradicts Nakayama's lemma.

(c) $\Rightarrow$ (a). By 1.1 it suffices to show that for $0 \neq x, y \in R$,

$$
R x \subseteq R y \text { or } R y \subseteq R x \text {. }
$$

By (3), $x \in J^{n}, x \notin J^{n+1}$ for some $n \geqslant 0$. Using (1) and (2), $R x+J^{n+1} / J^{n+1}$ is isomorphic to $J^{n} / J^{n+1}$. This forces $R x+J^{n+1}=J^{n}$.

As $J^{n}$ is finitely generated, $R x \subseteq J^{n}$, and $J$ is an ideal of $R$, we have as a consequence of Nakayama's lemma that $R x+J^{n+1}=J^{n}$ implies $R x=J^{n}$. Using an identical argument, we also have that $R y=J^{m}$ for some $m \geqslant 0$. Now depending on whether $n \geqslant m$ or $m \geqslant n, R y \supseteq R x$ or $R x \supseteq R y$.

1.7 Remark. Let $K$ be a field. Consider the ring of power series in one variable over $K$. Then the ideals of $K(x)=R$, are precisely the $R x^{n}, n=0,1, \ldots$, where each $R x^{n}=J^{n}, J=R x$. Hence by $1.6, R$ is an $l$-ring. This gives an example of a noetherian $l$-ring which is not artinian.

\section{Artinian $l$-rings}

A principal indecomposable projective module in an artinian ring $R$ is a projective module of the form $R e$ where $e$ is a primitive idempotent. An artinian ring is called left (right) generalized uniserial in case every principal indecomposable projective left (right) module has a unique composition series. Using these definitions we have the following proposition.

2.1 Proposition. A ring $R$ is an artinian l-ring if and only if it is a local left generalized uniserial ring.

Proof. $\leftarrow$ Suppose $R$ is a local left generalized uniserial ring. Since $R$ is local, this means that

$$
{ }_{R} R \supseteq J \supseteq \ldots \supseteq J^{n} \supseteq 0
$$

is the unique composition series for ${ }_{R} R$. Hence $R$ satisfies the conditions of $1.6(\mathrm{c})$ which implies that $R$ is an $l$-ring.

On the other hand, suppose that $R$ is an artinian $l$-ring. By $1.6(\mathrm{~b})$ the nonzero left ideals are precisely the $J^{k} \neq 0, k \geqslant 0$. Since $R$ is artinian, $J^{n}=0$ for some $n>0$. Therefore, since each $J^{k} / J^{k+1}$ is simple by $1.6(\mathrm{c})$,

$$
{ }_{R} R \supseteq J \supseteq \ldots \supseteq J^{n-1} \supseteq 0
$$

is the unique composition series for ${ }_{R} R$. This implies that $R$ is left generalized uniserial.

We investigate the conditions under which $l$-rings are $r$-rings when $R$ is an artinian ring. In view of 2.1 , this reduces to the question of when a local left generalized 
uniserial ring is right generalized uniserial. Rings which are both left and right generalized uniserial are called generalized uniserial. Local artinian rings which are generalized uniserial are precisely those artinian rings which are both an $l$-ring and an $r$-ring. Generalized uniserial rings have been studied intensively by a number of authors. They have the property that their modules can be represented as a direct sum of cyclics. Left generalized uniserial rings in general do not possess this property. It is therefore of interest to know when a left generalized uniserial ring is generalized uniserial. First we give an example of an $l$-ring which is not an $r$-ring by producing a left generalized uniserial local ring that is not generalized uniserial. This answers a question posed by Gluskin (1960), p. 303.

2.2 ExAmple. Let $F(t)$ be the field of rational functions over a field $F$. Let $R$ be the ring of all pairs $(f(t), g(t))$ where $f(t), g(t) \in F(t)$ with respect to componentwise addition and multiplication defined via

$$
\left(f_{1}(t), g_{1}(t)\right) \cdot\left(f_{2}(t), g_{2}(t)\right)=\left(f_{1}(t) f_{2}(t), f_{1}(t) g_{2}(t)+g_{1}(t) f_{2}\left(t^{2}\right)\right) \text {. }
$$

It can easily be shown that the radical $J=\{(0, g(t)) \mid g(t) \in F(t)\}$ and that $J^{2}=0$. Now note that for $g(t) \neq 0$ and $h(t) \in F(t)$

$$
\left(g^{-1}(t) h(t), 0\right) \cdot(0, g(t))=(0, h(t)) .
$$

Hence $R(0, g(t))=J$. As $(0, g(t))$ is an arbitrary nonzero element of $J$, we have that ${ }_{R} J$ is simple. Thus $R$ is left generalized uniserial. On the other hand, consider

$$
(0,1) R=\left\{\left(0, f\left(t^{2}\right)\right)\right\} ; \quad(0, t) R=\left\{\left(0, t . f\left(t^{2}\right)\right)\right\} .
$$

It is a straightforward computation to check that $(0,1) R$ and $(0, t) R$ are simple and that

$$
J_{R}=(0,1) R \oplus(0, t) R=F(t) \oplus F(t) .
$$

Thus $R$ is not right generalized uniserial. Hence $R$ is not an $r$-ring. The above example is a ring of the form $Q=R / J$ commutative with $J^{2}=0$ and $\operatorname{dim}\left(\left({ }_{Q} J\right) \cdot \operatorname{dim}\left(J_{Q}\right)=2\right.$. This type of ring provides examples of $l$-rings $(r$-rings) which are not $r$-rings ( $l$-rings) and has been studied extensively. For example, see Dlab and Ringel (1972).

We shall need the following observations which will be used in subsequent developments. For a ring $R$ to be generalized uniserial it suffices to show that $R / J^{2}$ is generalized uniserial (Nakayama (1940)). It can easily be shown that factor rings of $r$-rings are $r$-rings. The above remarks yield:

2.3 LeMma. Let $R$ be an artinian r-ring. Then $R$ is an l-ring if and only if $R / J^{2}$ is an l-ring.

We are now ready to prove the following theorem which gives sufficient conditions for an $l$-ring to be an $r$-ring. 
2.4 TheOREM. Let $R$ be a finite dimensional algebra over a field $K$. The following conditions are equivalent:

(a) $R$ is an l-ring.

(b) $R$ is a left generalized uniserial local ring.

(c) $R$ is an $r$-ring.

(d) $R$ is a right generalized uniserial local ring.

Hence $R$ is a generalized uniserial algebra if any one of the above conditions hold.

Proof. (a) $\Leftrightarrow$ (b) and (c) $\Leftrightarrow$ (d) by 2.1. We prove that (c) implies (a). That (a) implies (c) will follow by symmetry. We will assume that $J^{2}=0$, since by 2.3 it suffices to prove the theorem under those assumptions. Since $K$ is contained in the centre of $R$, the left and right dimensions of $\bar{R}=R / J$ over $K$ are the same. Set $n=\operatorname{dim}_{K}(\bar{R})$. Likewise,

$$
\operatorname{dim}_{K}\left({ }_{K} J\right)=\operatorname{dim}_{K}\left(J_{K}\right) .
$$

Suppose ${ }_{R} J=R s \oplus I$ where $0 \neq s \in J$ and $I$ is a left ideal such that $I \subseteq J$. By hypothesis $J_{K} \cong \bar{R}$ as $R$ modules and therefore as $K$ modules. Hence

$$
n=\operatorname{dim}_{K}(R s)+\operatorname{dim}_{K}(I)=n+\operatorname{dim}_{K}(I) .
$$

Thus, $\operatorname{dim}_{K}(I)=0$ which implies that $I=0$. Thus $R$ is left generalized uniserial, hence generalized uniserial.

\section{A non-noetherian $l$-ring}

In this section we shall give an example of an $l$-ring which is not noetherian. Consider the abelian group $Z_{\infty}^{p}$ consisting of all $p^{n}$ th roots of unity where $p$ is a prime and $n$ varies over all positive integers. Let $Z_{p} \cdot Z_{p}^{\infty}$ be the group algebra of $Z_{p}^{\infty}$ over the field $Z_{p}$. We will show that $Z_{p} . Z_{p}^{\infty}$ is a non-noetherian $l$-ring. This will be done by showing that the principal ideals are linearly ordered. Hence $Z_{p} . Z_{p}^{\infty}$ will be an $l$-ring by 1.1 . We begin by establishing a number of basic facts about $Z_{p} \cdot Z_{\infty}^{p}$.

Let $\varepsilon_{n}^{k}=\left(\varepsilon_{n}\right)^{k}$ where $1 \leqslant k \leqslant p^{n}$ and $\varepsilon_{n}$ denotes $\exp \left(2 \pi i / p^{n}\right)$, a primitive $p^{n}$ th root of unity for each $n \geqslant 0$. Let $x \in Z_{p}$. $Z_{\infty}^{p}$. Then if $x \neq 0$, there exists $t>0$ such that $x$ can be expressed as a sum of $t$ nonzero terms,

$$
x=\varepsilon_{s_{1}}^{k_{1}}+\ldots+\varepsilon_{s_{t}}^{k_{t}}
$$

where $s_{1} \leqslant s_{2} \leqslant \ldots \leqslant s_{t}$ and the $k_{i}$ 's and $s_{i}$ 's are not necessarily distinct.

Using the fact that for $n \geqslant s\left(\varepsilon_{n}^{k}\right)^{p^{s}}=\varepsilon_{n-s}^{k}$ and that $p \varepsilon_{n}^{k}=0$, we have

$$
x^{p^{n}}=\left(\varepsilon_{s_{1}}^{k_{1}}\right)^{p^{n}}+\ldots+\left(\varepsilon_{s_{t}}^{k_{t}}\right)^{p^{n}}=1+\ldots+1=t
$$

where $s_{1} \leqslant s_{2} \leqslant \ldots \leqslant s_{t} \leqslant n$. So $x$ is a non-unit or a unit depending on whether $x$ is a sum of a multiple of $p$ nonzero terms when expressed in the above form. 
Also the principal ideals generated by the elements

$$
1+(p-1) \varepsilon_{n} \text { for } n=1,2, \ldots
$$

form an increasing chain of ideals. For let $x=1+(p-1) \varepsilon_{m}, y=1+(p-1) \varepsilon_{n}$ where $m \leqslant n$. Thus $n=m+t$. So $y^{p^{t}}=1+(p-1) \varepsilon_{n-t}=1+(p-1) \varepsilon_{m}=x$.

Using the above results, we have

3.1 LEMMA. $Z_{p} . Z_{\infty}^{p}$ is a commutative non-noetherian ring whose maximal ideal consists of those elements which are a sum of $t$ nonzero terms of the form

$$
x=\varepsilon_{s_{1}}^{k_{1}}+\ldots+\varepsilon_{s_{t}}^{k_{t}}
$$

where $t=n p, n \geqslant 0$ and $s_{1} \leqslant s_{2} \leqslant \ldots \leqslant s_{t}$.

Now consider the group algebra $Z_{p} . Z\left(p^{n}\right)$ where $Z\left(p^{n}\right)$ is the group of $p^{n}$ th roots of unity, $p$ is a prime and $n$ is a positive integer. It is easily checked that $J\left(Z_{p} \cdot Z\left(p^{n}\right)\right)$ is a principal ideal generated by $1+(p-1) \varepsilon_{n}$. Thus $Z_{p} \cdot Z\left(p^{n}\right) / J^{2}\left(Z_{p} \cdot Z\left(p^{n}\right)\right)$ is a generalized uniserial local ring. So by 2.1 it is an $l$-ring. Now using the remark preceding 2.3 , we have that $Z_{p} . Z\left(\mathrm{p}^{n}\right)$ is an $l$-ring. This fact will be used in the proof of the following theorem.

3.2 TheOREM. Let $R=Z_{p} \cdot Z_{\infty}^{p}$ where $p>0$ is a prime. Then $R$ is an l-ring.

Proof. Let $x, y \in Z_{p} . Z_{\infty}^{p}$. Consider the following expressions for $x$ and $y$ :

$$
\begin{aligned}
& x=\varepsilon_{s_{1}}^{k_{1}}+\ldots+\varepsilon_{s_{v}}^{k_{v}}, \\
& y=\varepsilon_{t_{1}}^{j_{1}}+\ldots+\varepsilon_{t_{m}}^{j_{m}},
\end{aligned}
$$

where $s_{1} \leqslant \ldots \leqslant s_{v}, t_{1} \leqslant \ldots \leqslant t_{m}$. Let $n=\max \left(t_{m}, s_{v}\right)$. Then $x, y \in Z_{p} . Z\left(p^{n}\right)$. By the previous remarks $Z_{p} . Z\left(p^{n}\right)$ is an $l$-ring. Hence either

or

$$
Z_{p} \cdot Z\left(p^{n}\right) \cdot x \subseteq Z_{p} \cdot Z\left(p^{n}\right) y
$$

$$
Z_{p} \cdot Z\left(p^{n}\right) y \subseteq Z_{p} . Z\left(p^{n}\right) x .
$$

Thus it follows that $R x \subseteq R y$ or $R y \subseteq R x$.

\section{References}

V. Dlab and C. M. Ringel (1972), 'A class of balanced non-uniserial rings', Math. Ann. 195, 279-291.

L. M. Gluskin (1960), 'Ideals in rings and their multiplicative semigroups', Uspehi Mat. Nauk (N.s.) 1t, 141-148, translated in Amer. Math. Soc. Transl. 27(2), 1963, 297-304.

A. W. Goldie (1962), 'Non-commutative principal ideal rings', Arch. Math. 13, 213-221.

J. Lambek (1966), Lectures in rings and modules (Blaisdell, Waltham, Mass., U.S.A.).

T. Nakayama (1940), 'Note on uniserial and generalized uniserial rings', Proc. Imp. Soc. Japan 16, 285-289. 


\section{Trinity College and Universidad Nacional Experimental de Tachira Dublin, Ireland San Cristobal, Venezuela}

Current address:

Instituto de Matematica

Universidade Federal de Bahia

Caetano Moura, 99, Federação 40000

Salvador, Bahia

Brasil 